\title{
Targeted band gap creation using mixed sonic crystal arrays including resonators and rigid scatterers
}

\author{
E. Fuster-Garcia, V. Romero-García, and J. V. Sánchez-Pérez ${ }^{a)}$ \\ Centro de Tecnologías Físicas: Acústica, Materiales y Astrofísica, Universidad Politécnica de Valencia, \\ Cno. de Vera s/n, 46022 Valencia, Spain \\ L. M. García-Raffi \\ Instituto de Matemática Pura y Aplicada, Universidad Politécnica de Valencia, Cno. de Vera s/n, 46022 \\ Valencia, Spain
}

(Received 14 March 2007; accepted 22 May 2007; published online 13 June 2007)

\begin{abstract}
Sonic crystals are periodic structures that have acoustic band gaps centred at frequencies depending on the lattice constant of the array and on the direction of the incident acoustic wave. To eliminate this dependence, this work presents designed mixed structures constructed with rigid scatterers and resonators embedded in air. Specifically, balloons filled with a blend of air and helium were used as resonators, showing experimental evidence about the resonant behavior of an array formed with these balloons. As a result, the authors obtain full band gaps in a predetermined range of frequencies desired. (C) 2007 American Institute of Physics. [DOI: 10.1063/1.2748853]
\end{abstract}

Sound attenuation has become a standard topic of science and technology over the years. The traditional technique for reducing noise consists in increasing the thickness or the mass per unit area of the sonic shield materials used. However, one of the main disadvantages of this technique is the normally large size of the devices necessary to obtain suitable results.

In the last decade acoustic periodic composites based on heterogeneous materials, with a strong periodic modulation of the elastic constants between them, have been developed. These composite materials, usually called sonic crystals (SCs), have the property of creating spectral band gaps that do not allow acoustic wave propagation in a predetermined range of frequencies, depending on the periodicity of the array formed. Then the position of the band gap obtained depends on the direction of the incident wave on the SC. The sound attenuation effect is due to the multiple scattering generated inside these heterogeneous periodic materials and is the result of destructive interferences of scattered waves inside the gap. A great effort has been made to characterize these systems and to develop their technological applications. ${ }^{1-4}$ Specific research has recently been done to obtain band gaps in the low frequency range breaking the constant lattice dependence using resonators, the so-called locally resonant sonic materials. ${ }^{5-9}$ It has been proven that sonic band gaps can be obtained in several ranges of frequencies which do not depend on the periodicity of the crystal but on the resonance frequency of the resonators. It is thus possible to create an acoustic band gap for specific and relatively low frequencies, breaking the mass law.

On the other hand, some of us have recently proven the possibility of creating band gaps, with SCs made with rigid scatterers in air, in a predetermined range of frequencies. An optimization technique called genetic algorithms ${ }^{10}$ (GAs) together with the multiple scattering theory ${ }^{11,12}$ (MST) was used to achieve this purpose. The GA+MST method was previously used in the design of acoustical devices based on

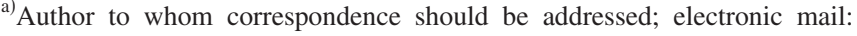
jusanc@fis.upv.es
}

SCs. ${ }^{13-16}$ In our case, the mechanism used was the creation of vacancies in a starting complete SC built with aluminum cylinders in air. We consequently obtained so-called quasiordered structures (QOSs). ${ }^{17}$

Nevertheless, the angular dependence of the SC in the acoustic attenuation obtained, i.e., the nonexistence of full band gaps, can still be a serious problem in constructing acoustic barriers or filters based on SCs with air such as host material. The use of resonators in these SCs might be one form of solution. Up to now, research about SCs using soft scatterers deployed in air focused on the use of balloons containing different gases as scatterers. Kushwaha and Halevi ${ }^{18}$ theoretically studied the behavior of threedimensional (3D) sonic crystals made with spherical balloons containing hydrogen gas and involved in air. In these systems the authors analyzed the band gap creation for three different 3D configurations as a function of the filling fraction, the latex wall thickness, and the pressure inside the balloons, explaining their appearance in terms of the multiple scattering theory.

In this letter we present evidence about the resonant behavior of an array of resonators (balloons containing a blend of air and helium) embedded in air. The experimental transmission results obtained suggest that there is no possibility of explaining the attenuation peaks observed as a function of Bragg's law or by the multiple scattering theory. Moreover, we have analyzed the acoustic behavior of mixed structures formed with both rigid cylinders and balloons in air. The results obtained show the creation of full band gaps in predetermined ranges of frequencies.

The experiments were performed in an echo-free chamber. In all cases, we measured the sound attenuation spectrum usually called the insertion loss. This parameter is the difference between the sound level recorded at the same point with and without the sample. We use rigid (aluminum) cylinders or resonators (balloons) as scatterers arranged in two triangular configurations with lattice constants $a=6.35$ and $12.7 \mathrm{~cm}$, respectively. The aluminum cylinders used have diameter $d=4 \mathrm{~cm}$ and length of $1 \mathrm{~m}$. The common balloons used in the experiments have a cylindrical shape, with 

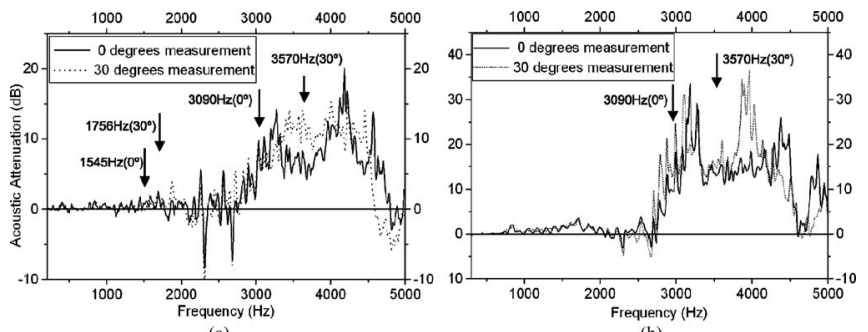

(a)

(b)

FIG. 1. Attenuation spectra of an array formed by five rows of ten balloons in triangular symmetry and for two incident directions $\left(0^{\circ}\right.$ and $\left.30^{\circ}\right)$. (a) Lattice constant $a=12.7 \mathrm{~cm}$ and (b) lattice constant $a=6.35 \mathrm{~cm}$.

$1 \mathrm{~m}$ length and diameters between 3.5 and $4.5 \mathrm{~cm}$. The variation of the diameter is due to their commercial origin that consequently involves differences in shape and small deformations. The balloons contain a standard blend of air and helium with density $\rho_{H}=0.9 \mathrm{~kg} / \mathrm{m}^{3}$ and an average of the inner pressure $p_{H}=140.000 \mathrm{~Pa}$. Moreover, we consider the latex wall thickness to be negligible compared to the diameter of the balloon. The samples are built up by hanging cylindrical elements on a frame. In order to keep the balloons in the vertical position, we tie down a little weight at their free extreme. This frame can rotate around the vertical axis, so one can explore any direction of the $k$ wave vector perpendicular to the cylinder axis. In particular, we made measurements along the two high-symmetry directions on the Brillouin zone $\left(\Gamma X-0^{\circ}\right.$ and $\left.\Gamma J-30^{\circ}\right)$ of the sample studied. A directional sound source B\&K 4204 emitting continuous white noise and a dual channel signal analyzer-type B\&K 2148 were used in all the experiments. The geometry used and the direction of the measurements analyzed are represented in Fig. 2.

First, we took measurements in order to verify the acoustic behavior of an array made only with balloons. We sought to prove its acoustic response by varying the filling fraction, the direction of the incident wave over the array, and the lattice constant. For this purpose we constructed an array of balloons formed by five rows of ten elements each and with two lattice constants $a=12.7 \mathrm{~cm}$ and $a=6.35 \mathrm{~cm}$. We measured the insertion loss along both $\Gamma X$ and $\Gamma J$ directions. In Fig. 1(a) we show the insertion loss for the array formed with the lattice constant $a=12.7 \mathrm{~cm}$. An attenuation peak around the same range of frequencies (from 2700 to $4600-4700 \mathrm{~Hz}$ ) can be seen for both incident wave directions. These peaks do not correspond to the second attenuation peaks in the $\Gamma X$ and $\Gamma J$ directions centred at $f_{0^{\circ}}=3090 \mathrm{~Hz}$ and $f_{30^{\circ}}=3570 \mathrm{~Hz}$, respectively. Moreover, the attenuation peaks corresponding to a typical behavior of an $\mathrm{SC}$, related to the periodicity of the SC (first Bragg peaks at the $\Gamma X$ and $\Gamma J$ directions centred at $f_{0^{\circ}}=1545 \mathrm{~Hz}$ and $f_{30^{\circ}}=1756 \mathrm{~Hz}$ ), do not appear in the spectrum. In Fig. 1(b) we show the results obtained for the lattice constant $a=6.35 \mathrm{~cm}$. Again, there is no difference between the spectrum in the $\Gamma X$ and $\Gamma J$ directions. Finally, the difference between the level of attenuation between both spectra [Figs. 1(a) and 1(b)] can be explained in terms of the variation of the filling fraction of the balloons in both samples. Note that the range of the attenuated frequencies is the same for the two lattice constants and for the two incident directions.

Although the existence of the attenuation peaks showed cannot be explained in terms of Bragg's law, the results obtained are compatible with a resonant phenomenon due to the

Downloaded 14 Jun 2007 to 158.42.64.9. Redistribution subject

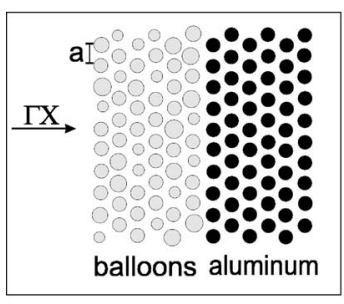

(a)

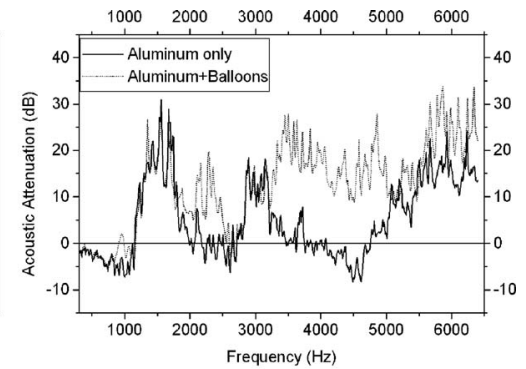

(b)
FIG. 2. Comparative acoustic attenuation spectra between an array of six rows of ten aluminium cylinders and a mixed structure of 12 rows formed by the previous one plus six rows of ten cylindrical balloons. In both cases, the lattice constants are $a=12.7 \mathrm{~cm}$, the incident direction is from left to right, and the measurements have been made along the $\Gamma X$ direction. (a) Schematic of the mixed structure analyzed. (b) Acoustic attenuation obtained for the two structures (SC and mixed structure).

nondependence of the attenuation peaks on the angle of the incident wave over the array and on the lattice constant. The resonant frequency due to the length of the balloons $(1 \mathrm{~m})$ would be less than $2700 \mathrm{~Hz}$, and then, the most important contribution in the attenuation peak observed will correspond to the oscillation of the balloons in a transversal plane. In short, we have demonstrated experimentally that arrays formed by balloons filled with gas with physical properties similar to that of the air act as an array of resonators against the theoretical results showed in Ref. 18.

In Fig. 2(a) we show a mixed structure formed by an array made with six rows of ten aluminum scatterers plus six rows of balloons, situated as the figure shows. The lattice constant of the mixed structure is $a=12.7 \mathrm{~cm}$. In Fig. 2(b) we show both the attenuation spectra measured at $0^{\circ}$ of the $\mathrm{SC}$ formed with rigid cylinders and the one corresponding to the mixed structure. It can be seen that the spectrum of the mixed structure shows the acoustic attenuation due to the SC with rigid cylinders plus the attenuation produced by the balloons due to the resonance phenomena. This fact means that the mixed structure allows us to get an enhancement of the attenuation band.

Finally, in order to obtain a full band gap in a predetermined range of frequencies, we constructed a mixed structure formed by one of the QOS proposed by Romero-García et al. $^{17}$ [QOS in Fig. 3(d)] plus balloons. The QOS chosen is formed by rigid (aluminum) cylinders and has been optimized to attenuate a range of frequencies from 1700 to $2300 \mathrm{~Hz}$ by means of the creation of vacancies in a complete SC. Their main characteristics are six rows of ten aluminium cylinders each and a lattice constant $a=6.35 \mathrm{~cm}$, which correspond to a Bragg frequency of $3090 \mathrm{~Hz}$. We probed the existence of a full band gap at a frequency range from 1700 to $2300 \mathrm{~Hz}$ in this QOS. The balloons have been placed at the site of the vacancies created, so the width of the mixed structure and the total number of elements are the same as the starting SC. Figure 3(a) shows the resulting mixed structure. In Figs. 3(b) and 3(c) we compare the acoustic attenuation spectra obtained at the two highsymmetry directions of the starting SC with those obtained only with the QOS. In both cases, the attenuation level increases when we put balloons in the place of the vacancies. In Fig. 3(d), one can see the large full band gap obtained in the predetermined range of frequencies chosen.

AIP license or copyright, see http://apl.aip.org/apl/copyright.jsp 


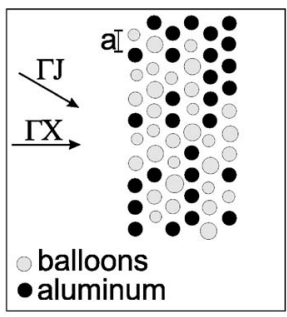

(a)

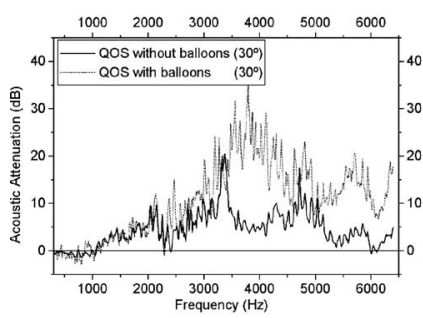

(c)

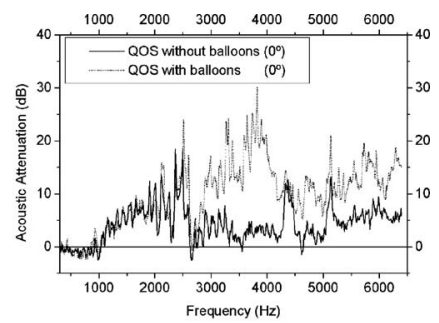

(b)

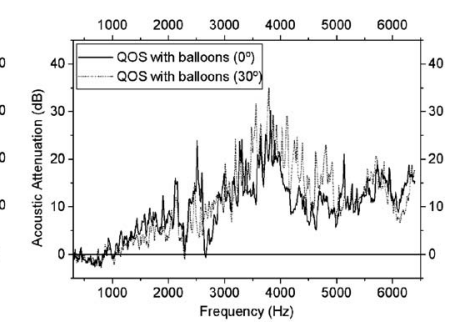

(d)
FIG. 3. Acoustic attenuation obtained with the mixed structures proposed. (a) Schematic of the mixed structure designed. (b) Attenuation spectra obtained for both structures, QOS with and without balloons, at $0^{\circ}$, (c) for the previous structures measured at $30^{\circ}$, and (d) for the mixed structure measured at $0^{\circ}$ and $30^{\circ}$.

We have demonstrated that it is possible to increase the attenuation band of the SCs made with scatterers embedded in air, using mixed structures of rigid scatterers and resonators filled with gas with physical properties similar to those of the air. The advantage of these mixed structures, compared to those formed only by resonators, is that we can choose both the range of resonant frequencies (in our case by means of the diameter of the balloons) and the range of multiple scattering frequencies creating vacancies using GA in an SC made with rigid cylinders with a reasonable lattice constant. The sum of both effects (resonance and multiple scattering) allows us to design structures with enhanced acoustic attenuation, creating a full band gap in a predetermined range of frequencies. Intensive research naturally needs to be done to develop the necessary technology to use these structures as acoustic barriers.

This work has been supported by Spain's Interministerial Science and Technology Commission (Contract No. 200603097) and FEDER and by the Generalitat Valenciana (Spain) under Grant No. GV/2007/191. The authors would also like to thank the $\mathrm{R}+\mathrm{D}+\mathrm{i}$ Linguistic Assistance Office at the Universidad Politécnica of Valencia for their help in revising this letter.

${ }^{1}$ J. V. Sánchez-Pérez, D. Caballero, R. Martínez-Sala, C. Rubio, J. Sánchez-Dehesa, F. Meseguer, J. Llinares, and F. Gálvez, Phys. Rev. Lett. 80, 5325 (1998).

${ }^{2}$ M. S. Kushwaha, Appl. Phys. Lett. 70, 3218 (1997).

${ }^{3}$ J. V. Sánchez-Pérez, C. Rubio, R. Martínez-Sala, R. Sánchez-Grandía, and V. Gómez, Appl. Phys. Lett. 81, 5240 (2002).

${ }^{4}$ F. Cervera, L. Sanchis, J. V. Sánchez-Pérez, R. Martínez-Sala, C. Rubio, F. Meseguer, C. López, D. Caballero, and J. Sánchez-Dehesa, Phys. Rev. Lett. 88, 023902 (2002).

${ }^{5}$ Z. Liu, X. Zhang, Y. Mao, Y. Y. Zhu, Z. Yang, C. T. Chan, and P. Sheng, Science 289, 1734 (2000).

${ }^{6}$ K. M. Ho, C. K. Cheng, Z. Yang, X. X. Zhang, and P. Sheng, Appl. Phys. Lett. 83, 5566 (2003).

${ }^{7}$ C. Goffaux, J. Sánchez-Dehesa, A. L. Yeyati, Ph. Lambin, A. Khelif, J. O. Vasseur, and B. Djafari-Rouhani, Phys. Rev. Lett. 88, 225502 (2002).

${ }^{8}$ C. Goffaux and J. Sánchez-Dehesa, Phys. Rev. B 67, 144301 (2003).

${ }^{9}$ M. Hirsekorn, P. P. Delsanto, N. K. Batra, and P. Matic, Ultrasonics 42, 231 (2004).

${ }^{10}$ J. H. Holland, Adaptation in Natural and Artificial Systems (The University of Michigan Press, Ann Harbor, MI, 1975).

${ }^{11}$ Z. Ye and Y. Chen, Phys. Rev. E 64, 036616 (2001).

${ }^{12}$ L. Sanchis, F. Cervera, J. Sánchez-Dehesa, J. V. Sánchez-Pérez, C. Rubio, and R. Martínez-Sala, J. Acoust. Soc. Am. 109, 2598 (2001).

${ }^{13}$ A. Hakansson, J. Sánchez-Dehesa, and L. Sanchis, Phys. Rev. B 70, 214302 (2004).

${ }^{14}$ L. Sanchis, A. Hakansson, D. López-Zanón, J. Bravo-Abad, and J. Sánchez-Dehesa, Appl. Phys. Lett. 84, 4460 (2004).

${ }^{15}$ A. Hakansson, F. Cervera, and J. Sánchez-Dehesa, Appl. Phys. Lett. 86, 054102 (2005).

${ }^{16}$ A. Hakansson, J. Sánchez-Dehesa, and F. Cervera, Appl. Phys. Lett. 88, 163506 (2006).

${ }^{17}$ V. Romero-García, E. Fuster, L. M. García-Raffi, E. A. Sánchez-Pérez, M. Sopena, J. Llinares, and J. V. Sánchez-Pérez, Appl. Phys. Lett. 88, 174104 (2006).

${ }^{18}$ M. S. Kushwaha and P. Halevi, J. Acoust. Soc. Am. 101, 619 (1997). 